\title{
Design of Monitoring System for Magnesium Alloy Sheet Four Roller Cold Rolling Mill
}

\author{
Fan Liping \\ College of Environment and Safety Engineering \\ Shenyang University of Chemical Technology \\ Shenyang, China \\ e-mail:flpsd@163.com
}

\author{
Han Rong \\ College of Information Engineering \\ Shenyang University of Chemical Technology \\ Shenyang, China \\ e-mail:1032800961@qq.com
}

\begin{abstract}
This paper designs a dynamic monitoring system applied to four cold rolling mill for Magnesium alloy sheet The system select Siemens WinCC6.0 as Monitoring interface and Siemens S7300PLC as lower computer for data communication and dynamic monitor. The main interface of the monitoring system is designed, and the fault alarm interface and communication between PC and PLC is implemented. Engineering practice shows that the monitoring system has many advantages of fast response and high reliability. It can keep the rolling process running well.
\end{abstract}

Keywords-rolling mill; magnesium sheet; PLC; monitoring; fault alarm

\section{INTRODUCTION}

With the rapid industrial development, Magnesium alloy sheet has a wide range of applications in the automotive industry, light industry, household appliances, construction, and so on. In the production process of magnesium alloy sheet, control system is necessary to monitor the operating status. Good performance of the monitoring and control system for a rolling mill is a guarantee of good product quality of magnesium sheet. In this paper, a PLC control and monitoring system is designed for the rolling mill system. The system S7300PLC is used as the main hardware control device to control the main drive motor system and the right and left auxiliary drive system, and is used as the process monitoring system. The real-time communication between WinCC and PLC is achieved by industrial Ethernet.

\section{MAGNESIUM ALLOY SHEET FouR ROLL COLD ROLLING MILL PROCESS DESCRIPTION}

The production process of magnesium alloy sheet is a process that magnesium workblank rolls back and forth by the rolling mill till final turns to the finished product. A set of basic magnesium alloy sheet rolling system contains transporting steel coils / unwinding device, the main mill, before and after crimping machine unloading car, delivery devices and hydraulic and lubrication stations. The cold rolled material is supplied by the hot rolling mill. Before the cold rolling process, the hot rolled magnesium alloy sheet should be pickled to remove magnesium oxide so as to make the surface of the magnesium alloy sheet smoothly and to make sure that the rolled product smoothly. Magnesium alloy sheet hot rolled pickled reversible after rolling an odd passes on the reversible rolling mill, finally get the desired thickness of the cold rolling Magnesium alloy sheet.

\section{CONTROL SyStem NETWORK TOPOLOGY DESIGN}

Magnesium alloy sheet mill PLC control system consists of a master station (Siemens S7-300PLC) and two slave sites, master site and the slave sites using the PROFIBUS DP to data exchange, network topology shown in Fig .1.

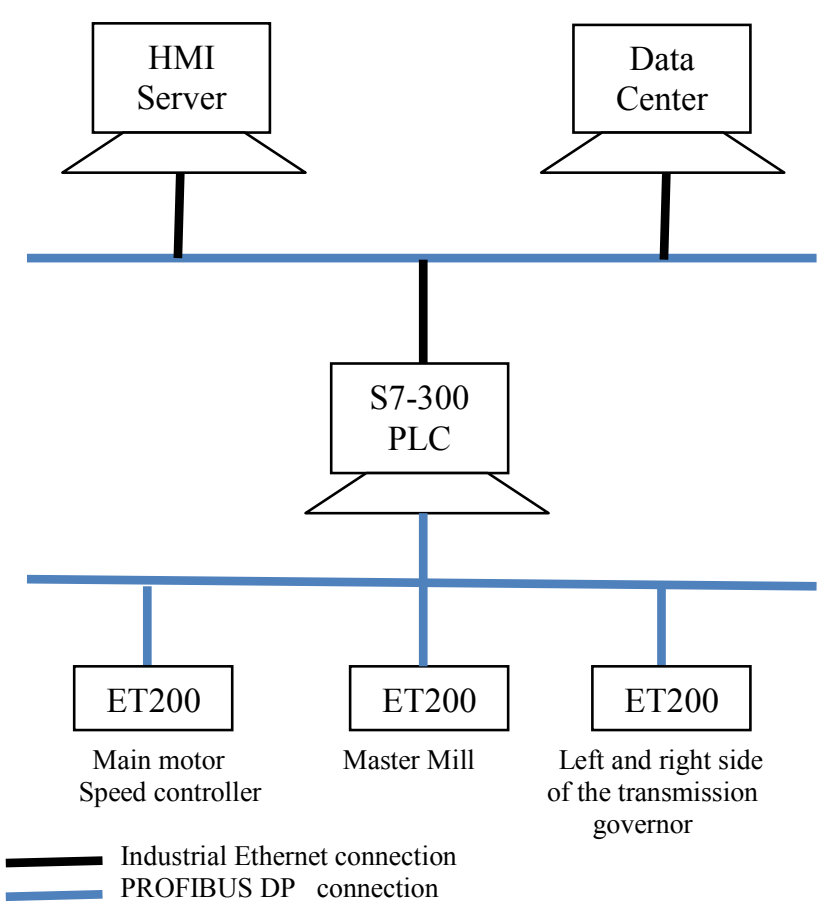

Figure 1.Network structure

\section{Windows CONTROL CENTER INTRODUCTION}

Siemens SIMATIC Windows Control Center windows control center is HMI / SCADA software. in 1996 to enter the world industrial control configuration software market, when it was named the best American Control Engineering magazine HMI software, in the shortest possible time, it becomes the third successful SCADA system in the world range, while in Europe, it became the undisputed first .

As the fully integrated automation system of SIMATIC important part, windows control center ensures connection with SIMATIC S5 and S7 series PLC 
convenience and efficient communication. windows control center and STEP7 integrate closely shorten the development cycle of the project.

Windows Control Center as an important part of the basic automation system for setting various data control system, display, fault alarm, online commissioning operation and maintenance of appropriate equipment, play an important role.

\section{CONTROL SyStem SCREEN DESIGN}

\section{A. The Main Interface Of Monitor Design}

In the system monitor screen, you can see the entire monitor screen of Magnesium alloy sheet rolling control system, it includes: Field parameters display screen, the display changes caused by the main parameters such as temperature, rolling force, current and voltage, and other major factors, there are eight button main interface below the main interface monitor, the main fault alarm button, hydraulic station button, and lubrication station buttons, rolling schedule buttons, etc, these buttons are linked to the corresponding monitoring interfaces, the operator can easily monitor the interface between the various switching to view the mill rolling process parameters in order to facilitate in time. monitoring interface as shown in Figure2.

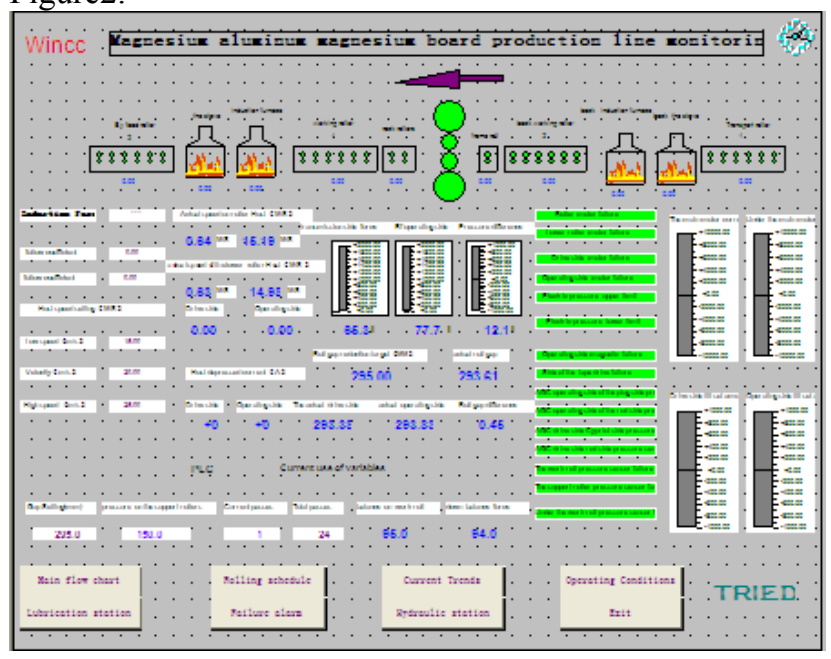

Figure 2. Monitor interface

Before rolling production, as shown in Fig .2, according to the different production need to enter the appropriate parameter values, it major to prepare for the production process. Windows Control Center put the corresponding values transferred to the PLC, these values will determine the production process what uses, such as temperature, rolling force, Rolling speed and so on.

\section{B. Error Alarm Interface Design}

Fault alarm interface includes a fault indicator, alarm acknowledgment button, alarm text, voice alarm, alarm records and so on, PLC transmitted to the host computer by detecting and diagnosing the fault signal .there is a corresponding alarm indicator light flashes and prompted alarm sounds in fault alarm screen.

Motor state represented by a specific color, green represents motor is running while red represents motor error. Fault alarm screen as shown in Fig .3.

PLC program are as follows:

\begin{tabular}{ccc} 
A & \multicolumn{2}{c}{ "M100.1” } \\
A & L & 0.0 \\
A & L & 0.0 \\
A & "M0.1" & \\
\multicolumn{4}{c}{ "M0.2" }
\end{tabular}

A "M0.3"

A "M0.4"

$=\quad \mathrm{M} \quad 20.1$

A $\quad$ L $\quad 0.0$

AN "M1.1"

AN "M1.2"

AN "M1.3"

AN "M1.4”

$=\quad \mathrm{M} 3.0$
// Register is cleared

// \# 1 motor has been closing of the upper Working roll

// bearing roller 2 \# motor has been closing of the upper supporting roller

// 3 \# motor has been closing of the lower working roller

// 4 \# motor has been closing of the lower supporting roller // All motors have been closing

// \# 1 motor error of the upper working rolling

// 2 \# motor error of the upper supporting roller

// 3 \# motor error of the lower working roller // 4 \# 1 motor error of The lower supporting roller // All motors without errors

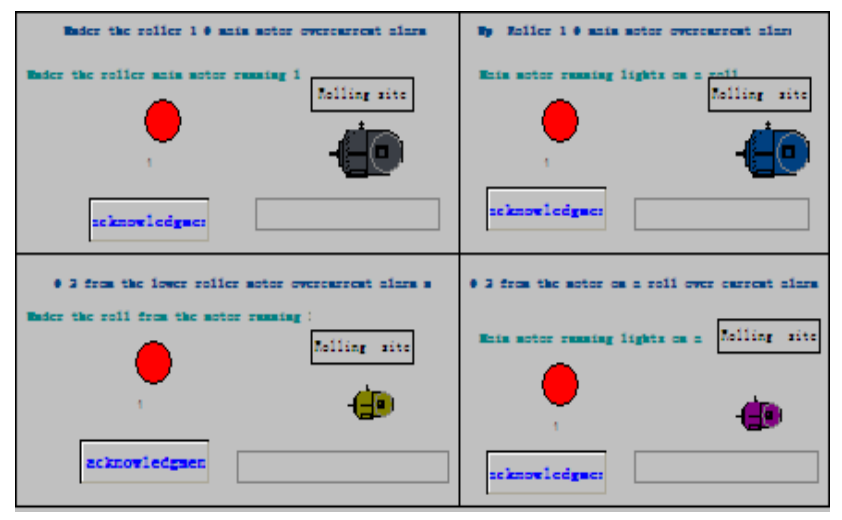

Figure 3.Alarm interface

Alarm records define what kind of alarm displayed, alarm contents, Fault location. Runtime of alarm records is mainly responsible for monitoring process values, control alarm output, alarm management confirmation. Figure 4 is picture of the alarm records in each pump, motor over current, pressure overload on the support roller alarm records. The red background indicates fault yellow background color indicates the amount of oil is running out of pump while the blue background color indicates the alarm status has been confirmed.

Number Fault text $\quad$ Fault location 


\begin{tabular}{|l|l|l|}
\hline 2 & Roller 2 \# Motor overcurrent & Thermal relay R2 \\
\hline 2 & Roller 2 \# Motor overcurrent & Thermal relay R2 \\
\hline 14 & Pump error & Hydraulic pump motor \\
\hline 14 & Pump error & Hydraulic pump motor \\
\hline 14 & Pump error & Hydraulic pump motor \\
\hline 3 & Under the roller 1 \# motor overcurrent & Thermal relay R3 \\
\hline 4 & Lower roller 2 \# Motor overcurrent & Thermal relay R4 \\
\hline 2 & Roller 2 \# Motor overcurrent & Thermal relay R2 \\
\hline 1 & \#1 motor overcurrent & Thermal relay R1 \\
\hline 1 & \#1 motor overcurrent & Thermal relay R1 \\
\hline 7 & The low roll pressure overload & 3 \# Pressure Sensors \\
\hline 7 & The low roll pressure overload & 3 \# Pressure Sensors \\
\hline 6 & Pressure overload on the support roll & 2 Pressure Sensors \\
\hline 14 & Pump error & Hydraulic pump motor \\
\hline 2 & Roller 2 \# Motor overcurrent & Thermal relay R2 \\
\hline 2 & Roller 2 \# Motor overcurrent & Thermal relay R2 \\
\hline 4 & Lower roller 2 \# Motor overcurrent & Thermal relay R4 \\
\hline
\end{tabular}

Figure 4.Error records interface

\section{COMMUNICATION CONFIGURATION}

The cold rolling mill of the Magnesium alloy sheet rolling system master Siemens S7- 300 PLC, including two ET200 slave station, two speed governors are the master motor governor and transmission governor system. Main station and slave station communicate by PROFIBUS-DP. Communicate with Ethernet between PC and PLC. Communication between PC and PLC configuration as the following steps: First, create a Windows control center project, protocol driver in the Tag Manager (Tag Management) based on your chosen selection communications and select the channel unit so that the channel unit Configuring a logical connection. After setting up the logical connection nodes, the network address (network address must be the same as setting PLC) and other parameters, then established communication link between PC and PLC: Second, communication connection has been established and then to define variables or variable group. Each variable has three settings needed: variable names, data types, variable address, in which the most important is the variable address, It has established a certain relationship between the variables and the corresponding address of PLC. The need for data communication between the PLC and Windows Control Center first to defined external variables to complete the data communication between the PLC and Windows Control Center so you can making the monitor screen base on element or elements of the graphics library graphics editor (Graphics Editor), the external variable element connected with each object is equivalent to each object connected with field devices to realize purpose of monitoring and controlling objective of field equipments.

\section{Windows CONTROL CENTER AND PlC S7-300 COMMUNICATION IMPLEMENTATION}

Industrial Ethernet is part of the management-level and unit-level SIMATIC network for transmission of large amounts of data and long-distance communication. On the physical connection, industrial Ethernet transmission media can be a coaxial cable, twisted pair, fiber optic and wireless communications.

First of all hardware, PLC station is connected to the Ethernet via Industrial Ethernet CP (CP343-1), PC is connected to the Ethernet via NIC. In order to reduce interference, so use shielded twisted pair.

PLC must download hardware configuration to the PLC by the MPI programming adapter (otherwise CP3431 not be used) at first.

The channel of unit use TCP / IP protocol. System Configuration CP343-1 Ethernet communication module for the PLC, System Configuration CP343-1 Ethernet communication module for PLC system, Communication configuration of SIMATIC Windows Control Center and S7 PLC automation system include the two methods: one is independent configuration mode that is communication between them is done through communication channels, communication does not require software in SETP7 configuration between them, you only need to set communication parameters on the windows control center configuration software and on the computer as a server set the appropriate network connection, create and configure the same way as the industrial MPI, if do this will be connected to the automation system windows control center S7PLC; another way is to integrate configuration, which uses Totally Integrated Automation framework STEP7 to manage windows control center project, in this way do not configuration variables, and defined variables and communication parameters in STEP7 can be transmitted to the windows control center project directly, in this way the project configuration tasks can be reduced more than half and can reduce configuration errors.

The need of Windows Control Center Variable Manager to add "SIMATIC S7 Protocol Suite.chn" driver either windows control center and SIMATIC S7-300PLC communication either way. This method is applicable to the management and field level communications, in favor of large amounts of data exchange, making the entire system with high reliability, speed up, and openness and so on. Specific steps are as follows:

The first step: under the STEP7 hardware configuration, configuration the Ethernet communication module IP addresses and masks, Install the CP-1613 newsletter template on windows control center and install CP343-1 / CP443-1 newsletter template on the PLC.

Step Two: Set the IP address and communication testing, ensure that the IP address of the computer and the PLC Ethernet communication module IP address is same network, and then use the PING command to test the Ethernet network is normal communication. Connect the Windows Control Center and PLC to the Ethernet by switcher.

Step Three: Add driver program and set system parameters: Open windows control center project Tag management- SIMATIC S7 PROTOCOL SUITE-TCP / IP and right-click pop-up menu, set system parameter, the logical device for CP-TCPIP.

Step Four: Set PG / PC Interface access mode under Windows operating system control panel CP-TCP/IP.

Step Five: Add the channel and the connection settings: Open the windows control center project Tag Management - SIMATIC S7 PROTOCOL SUITE -TCP / IP, right-click 
pop-up menu of new driver connection, and then set the communication module IP address and CPU slot number.

\section{COMMUNICATION DEBUGGING}

The Windows Control Center of the upper machine and the lower machine PLC connection to realize the main purpose is to verify the field equipment and industrial computer the communication link is normal. For example: the host Windows Control Center sends out the control command, whether the field equipment is able to correctly apply for, or the upper machine display the value whether should be relative and actual.

The upper computer sends the digital quantity control instruction to the lower machine by windows control center internal function or the $\mathrm{I} / \mathrm{O}$ domain. when the variable assignment is 1 , the variables corresponding to the PLC relay, Also in the SIMATIC Manager variable table status bit (Status value) can observe the color change.

The lower machine industrial site acquisition digital data directly from the DI module PLC Road input.

S7-300PLC analog signal channel is generally $0-10 \mathrm{~V}$ or $4-20 \mathrm{~mA}$. When the upper computer and field sensor numerical display numerical inconsistencies, can use the universal meter to measure the analog voltage or current signal input or output channel, or observe the numerical SIMATIC Manager variable table state bits to judge the position of communication error.

\section{CONCLUSION}

Taking Windows Control Center as monitoring software of host computer and S7-300PLC as the lower machine for rolling mill system can completely meet the requirement of Magnesium alloy sheet. This monitoring system HMI PC interface is friendly, the function is perfect, the whole monitoring system has been put into practical application, stable and reliable operation.

\section{REFERENCES}

[1] MA Guo-hua. Monitoring configuration software and its application [M] Beijing: Tsinghua University Press, 200

[2] ZHANG Xiang-jun , ,JIN Long-guo, YU Hu-min. Application of WinCC and S7 -300 PLC in control system of a reversible experimental four high mill[ J]. Metallurgical Industry Automation, 2002, 26(4) : $45-47,66$

[3] HE Hua. Layman Siemens Windows Control Center V6 [M] Beijing: Beijing University of Aeronautics and Astronautics, University Press, 2005: 5-10.

[4] SUN Yi-kang, TONG Chao-nan, Cold-rolled production automation technology [M]. Beijing: Metallurgical

[5] MAO Linkage. Methods S7 -300 series PLC and Configuration Software Windows Control Center achieve communication [J] International Mechatronics Technology, 2006, 9 (4): 32-34.

[6] Chen Ping Field bus and industrial control network technology [M]. Beijing: Electronic Industry out. Press, 2008

[7] Ding Xiu 1 rolling process automation [M] 1 Beijing: Metallurgical Industry Press, 1986

[8] XU Hua based pumping station automation control system Windows Control Center configuration software [J]. Coal Mining Machinery, 2013.Vol 34 No. 04: 244-246.

[9] Yan Yin-fu monitoring configuration software and PLC Starter [M]. Beijing: People's Post published

[10] PI Zhuang-xing design and application examples of Programmable Logic Controller [M]. Beijing: Machinery Industry Press, 2000. 